\title{
Subclinical hypothyroidism and mortality
}

Subclinical hypothyroidism is associated with increased all-cause and cardiovascular mortality, report researchers from Taiwan. "The associations of subclinical thyroid disorders and mortality have been studied in the literature but with controversial conclusions," explains senior researcher Kuo-Chin Huang of the National Taiwan University Hospital. "We have data from a large cohort collected from nationwide Health Screening Centers in Taiwan in 1998-1999. Using that cohort, we previously demonstrated the associations of betel nut chewing and BMI with mortality. We analysed the associations between subclinical hypothyroidism and mortality in this latest study."

The 115,746 study participants were aged $\geq 20$ years and had no history of thyroid disease. Their baseline TSH and total $\mathrm{T}_{4}$ levels were measured at screening: 1,841 participants $(1.6 \%)$ had subclinical hypothyroidism, defined as TSH levels 5.0-19.96 mIU/l and normal total $\mathrm{T}_{4}$ levels, whereas 113,905 were euthyroid. Baseline data on cardiovascular risk factors were also collected.

3,669 deaths occurred during the 10 years of follow-up, of which 680 were due to cardiovascular disease, according to the death certificates. Subclinical hypothyroidism was associated with increased all-cause mortality (relative risk [RR] 1.30, 95\% CI 1.02-1.66) and cardiovascular mortality (RR 1.68, 95\% CI 1.02-2.76), after adjustment for age, sex, BMI, diabetes mellitus, hypertension, dyslipidaemia, smoking, alcohol consumption, betel nut chewing, physical activity, income and education level.

Mark Vanderpump from the Royal Free London NHS Foundation Trust, who was not involved in this study, expresses some reservations about these results. "A wide TSH range was chosen to define subclinical hypothyroidism. Furthermore, the cohort had a surprisingly low prevalence of mild thyroid failure (1.5\%) compared to other cohorts."

He notes other study limitations, also acknowledged by the researchers, including a lack of measurement of free $\mathrm{T}_{4}$ levels or antithyroid antibodies, preventing ascertainment of the aetiology of thyroid failure. In addition, the researchers did not collect data on whether participants received follow-up thyroid function tests or levothyroxine therapy.

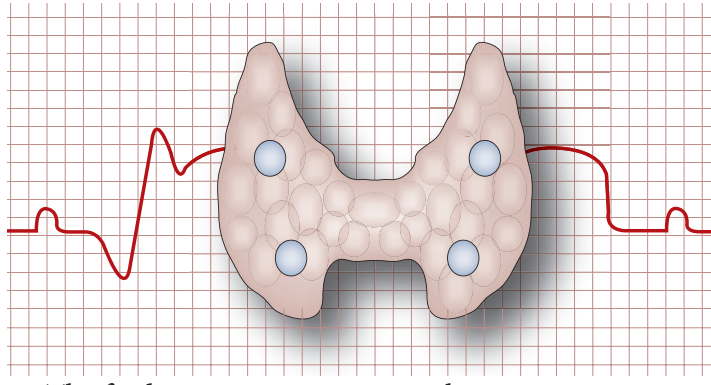

The findings suggest to Huang that screening for and follow-up of subclinical hypothyroidism is important, especially in elderly patients. "Taiwan is rather conservative in the treatment of subclinical disorders," he comments. "We would like to conduct studies to identify a suitable regimen for treating subclinical hypothyroidism."

Whether treating subclinical hypothyroidism would reduce cardiovascular and all-cause mortality remains unclear, Vanderpump notes. "Only a randomized controlled trial of levothyroxine intervention to normalize TSH will establish whether correction of early thyroid failure is associated with an improved cardiovascular outcome," he says.

\section{Carol Wilson}

Original article Tseng, F.-Y. et al. Subclinical hypothyroidism
is associated with increased risk for all-cause and
cardiovascular mortality in adults. J. Am. Coll. Cardiol.
doi:10.1016/j.jacc.2012.03.047

